\title{
Localised Pigmented Villonodular Synovitis Presenting As Anterior Knee Pain
}

\author{
JH Goh, MD, S Saravanan*, MS Ortho, WM Ng, MS Ortho, LM Looi**, FRCPath, R Ali, FRCS \\ Department of Orthopaedic Surgery, University Malaya Medical Centre, Kuala Lumpur, Malaysia \\ *Department of Orthopaedic, Teluk Intan Hospital, Teluk Intan, Malaysia \\ **Department of Pathology, University Malaya Medical Centre, Kuala Lumpur, Malaysia
}

\begin{abstract}
We report a case of a 21 years old female who presented with a history of anterior knee pain for previous 3 months. Pain was localized to the anteromedial aspect of the left knee and aggravated by flexion. Clinical examination revealed a $2 \times 2$ $\mathrm{cm}$ painful lump on the anteromedial aspect of the left medial condyle with no effusion. Magnetic resonance imaging revealed an anterosuperior tear of the medial collateral ligament. The patient subsequently underwent left knee arthroscopic examination. Two yellowish pedunculated masses arising from the anteromedial portion of the synovium were discovered and completely excised. Histopathological examination of the excision biopsy revealed pigmented villonodular synovitis (PVNS) with marked central necrosis. One year post excision, she is well with no signs or symptoms of recurrence. This case highlights an uncommon cause of anterior knee pain. Localized PVNS typically presents with mechanical symptoms, however, pain could arise from pedicle torsion and necrosis.
\end{abstract}

Key Words:

Pigmented Villonodular Synovitis, Anterior Knee Pain, Synovitis, Arthritis

\section{INTRODUCTION}

Anterior knee pain in adolescents is a common problem. The pain may arise from numerous anatomical structures found at the anterior aspect of the knee. Patellofemoral maltracking is the most common cause of anterior knee pain ${ }^{1}$. Other common causes are Hoffa syndrome that is caused by infrapatella fat pad impingement and plica syndrome caused by inflammation and irritation of the knee plica.

Pigmented villonodular synovitis (PVNS) is a rare benign proliferative disorder of unknown aetiology, more common in males and mainly affecting synovial joint bursae and tendon sheaths. First described by Jaffe et al in 1941, PVNS may be difficult to diagnose, meaning that delay in diagnosis is not uncommon ${ }^{2}$. PVNS is usually monoarticular and typically affects the knee. The lesion is usually pedunculated or less frequently sessile in appearance and is found in the meniscocapsular junction, the intercondylar notch region, the anterior tibial eminence or the lateral recesses ${ }^{1}$. It is also seen in the fat pad, medial femoral condyle, on the medial patellar synovial plica and inside popliteal cysts but is extremely rare in the posterior compartment of the knee.

\section{CASE REPORT}

A 21 year old girl presented with a complaint of left knee pain for the previous 3 months. The pain was localized to the anteromedial aspect of her left knee, aggravated by knee flexion and associated with a small painful lump on the anteromedial aspect of her left medial femoral condyle (Figure 1) which moved from front of the medial condyle to the side of the condyle on knee flexion. There was no history of trauma, fever or any other constitutional symptoms. The patient worked in a factory and needed to sit or walk most of the time in her employment. There were no relevant past medical history.

On examination, a tender lump was palpable on the anteromedial aspect of the left medial condyle with no effusion. It measured around $2 \mathrm{~cm}$ by $2 \mathrm{~cm}$ and attached to the tissues superior to lesion. The lump was firm and mobile to some extent. It was not palpable or visible when the knee was flexed and the tenderness moved to the medial aspect of the medial femoral condyle with such flexion. Position and tracking of patellar were normal and the patellar grinding test was normal. Palpation of joint lines was non-tender except around the painful lump and all ligaments were intact. Plain radiograph of the left knee was normal and magnetic resonance imaging of the knee showed an anterosuperior tear of the medial meniscus with a partial tear of the deep medial collateral ligament.

Knee arthroscopy revealed a yellowish pedunculated synovial mass (Figure 2) with minimal synovitis and no loose bodies were found. The ligaments and meniscus were normal. There was no cartilage damage or presence of 


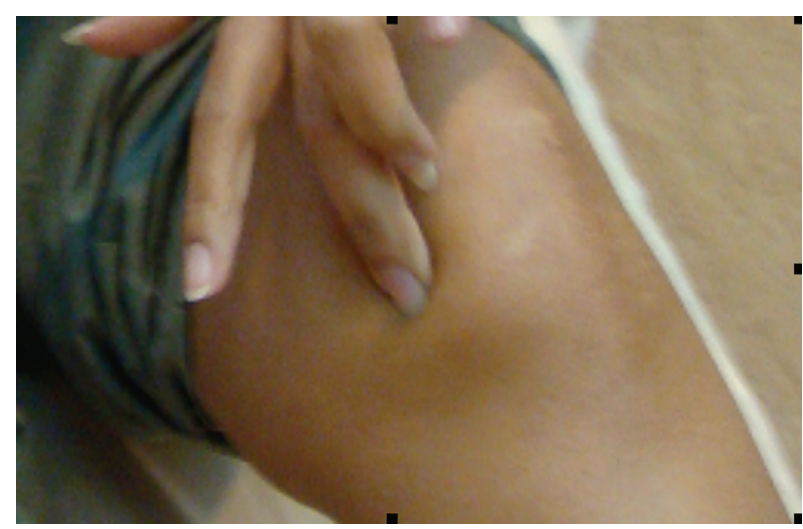

Fig. 1: Patient showing the exact site of pain and lump.

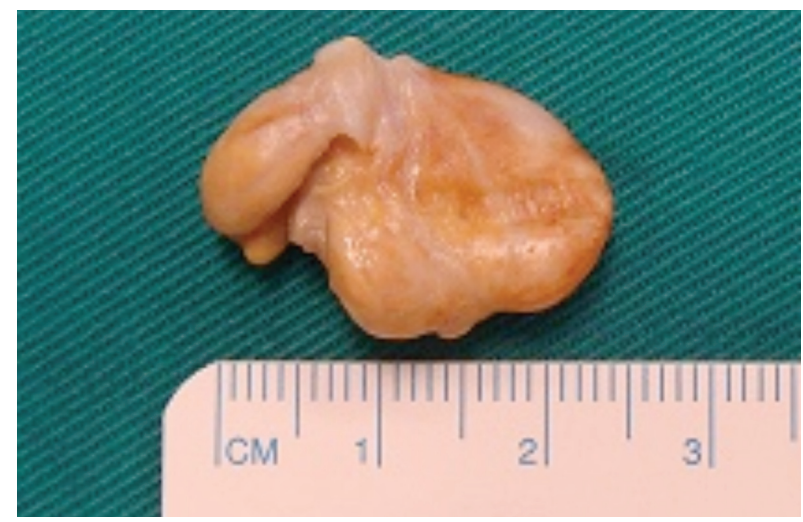

Fig. 3: Picture showing the lump and the measurement.

inflamed knee plicae. The masses were excised and sent for histopathological examination (Figure 3). Sectioning of the mass showed a nodule of hyaline necrotic material surrounded by inflamed synovium containing macrophages and occasional giant cells (Figure 4). Dystrophic calcification and some hemosiderin-laden macrophages were noted in the necrotic area. No malignant cells were detected. These findings resulted in a diagnosis of localized PVNS with central necrosis. Post-operatively the pain reduced gradually and the patient is now pain free.

\section{DISCUSSION}

The exact aetiopathology of PVNS is unknown. Incidence of localized PVNS is reported to be nine in one million, is more common in females and mainly affects the small joints. When localized PVNS affects large joints, $80 \%$ involve the knee. Patients usually present with mechanical symptoms and rarely with pain. However, Jaffe suggested that PVNS is an inflammatory reactive process with a proliferation of histiocytic cells ${ }^{2}$. There two distinct types of PVNS described, diffuse and localized, vary in clinical presentation and prognosis. Diffuse PVNS is more common, mainly affects large joints and is clinically characterized by synovitis, chronic diffuse pain with swelling and restricted joint movements.

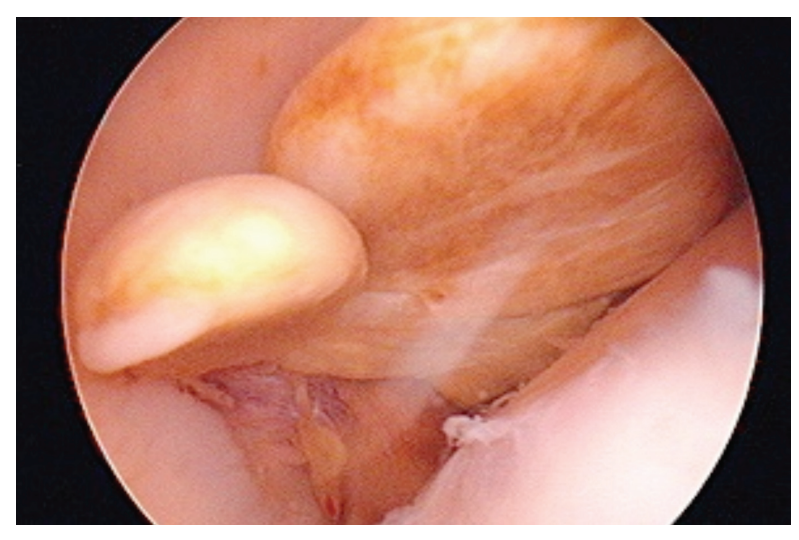

Fig. 2: Arthoscopic picture showing the pedunculated yellowish swelling.

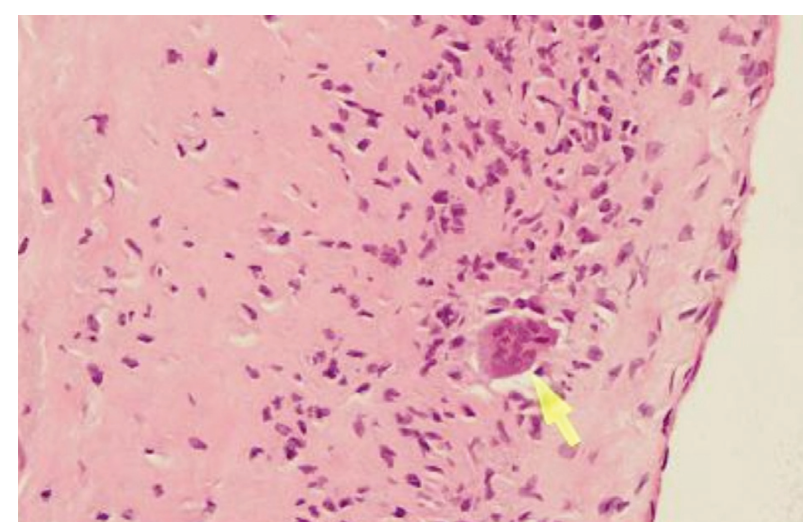

Fig. 4: Picture showing a multinucleated giant cell (yellow arrow), lymphocytes and synovium lining.

In the present case, pain is the predominant symptom. The presence of marked central necrosis in histopathological examination suggests torsional necrosis of the pedunculated mass. Therefore, localised PVNS may remain asymptomatic for many years until the pedicle is subjected to torsional necrosis, as reported by Howie ${ }^{3}$.

The range of differential diagnoses of anterior knee pain can vary from meniscal lesion, loose body and synovial plica to patellofemoral pain. Some cases of localised PVNS may result in cortical erosion but in the present case, the knee radiograph was normal. Magnetic resonance imaging is very sensitive but lacks specificity. Definitive diagnosis of localised PVNS depends on histological examination characterised by proliferation of synovial tissue with villi or synovial nodules, hemosiderin deposits, fibroblasts and possibly giant cells with fibrosis and hyalinization. These features (in addition of marked central necrosis) were demonstrated in the excision biopsy specimen of the current patient.

The treatment of choice for localized PVNS in the knee is complete excision either through open or arthroscopic surgery. Flandry presented the first case of successful arthroscopic resection of a localised PVNS in 1986 and this procedure has become more popular especially for smaller lesions ${ }^{4}$. The recurrence rate for localized PVNS post excision is negligible, as opposed to $45 \%$ in diffused PVNS. 


\section{REFERENCES}

1. Fulkerson. John P and Arendt EA. Anterior Knee Pain in Females. Clin Orthop 2000; 372. 69-73.

2. Jaffe HL, Lichtenstein L and Sutro CJ. Pigmented villonodular synovitis, bursitis and tenosynovitis. Arch Pathol. 1941; 1: 73165.

3. Howie CR, Smith GD, Christie J, Gregg PJ. Torsion of localized pigmented villonodular synovitis of the knee. J Bone Joint Surg Br, 1985; 67: 564-566.

4. Flandry F, Hughston JC. Pigmented villonodular synovitis. J Bone Joint Surg Am. 1987; 69: 942-9. 